\title{
Rückschau und Ausblick
}

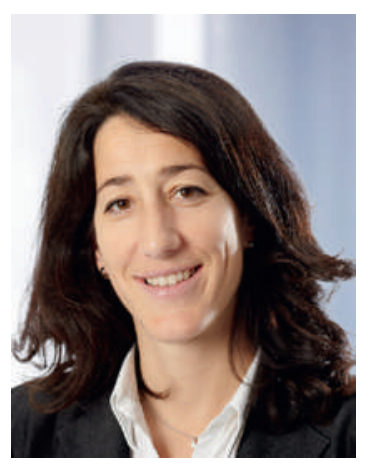

Die Weihnachtszeit ist für jeden von uns Anlass, das ausklingende Jahr Revue passieren zu lassen, aber auch unsere Erwartungen und unsere Hoffnungen für die kommenden Monate zu überdenken.

Wenn wir also Bilanz ziehen, werden wir uns fragen, ob wir der gewählten Richtung treu gefolgt sind und ob wir zur Erreichung der gesteckten Ziele wirklich alle erforderlichen Mittel eingesetzt haben. Wir werden an die Zufälle des Lebens denken, die uns möglicherweise von unserem Weg abgebracht und unsere Aufgabe dadurch erschwert oder auch erleichtert haben.

Für das Generalsekretariat der FMH gehört das Jahr 2012 nicht zu den einfachsten. Es steht für Veränderungen, Abwesenheiten, Austritte, aber auch für Erneuerung, Projekte, Visionen. Die Bilanz ist positiv. Die ersten Schritte der Restrukturierung und die Einführung der ersten neuen Führungsprozesse waren insbesondere durch den Wechsel einiger Vorstandsmitglieder und die neue Ressortverteilung geprägt. Für die Departemente des Generalsekretariats bedeutete das Jahr 2012 die Umsetzung verschiedener Projekte bzw. die Verschiebung anderer auf das kommende Jahr. Für mich war das Jahr 2012 ein Jahr der Führung aus der Ferne, mitgetragen von einem fantastischen Team, das mit viel Engagement bei der Sache war. Ich danke an dieser Stelle allen Mitarbeiterinnen und Mitarbeitern des Generalsekretariats für ihren gros-

\section{Unseren Service gegenüber den FMH-Mitgliedern können wir nur weiter verbessern und ihre Interessen ideal vertreten, wenn wir noch leistungs- fähiger werden und die begonnene Kosten- optimierung vorantreiben.}

sen Einsatz. All jenen, die die Notwendigkeit des Generalsekretariats und die unternommenen Anstrengungen zur Verbesserung seiner Strukturen und seiner Abläufe in Frage stellen, möchte ich Folgendes antworten: Unseren Service gegenüber den FMH-Mitgliedern können wir nur weiter verbessern und ihre Interessen ideal vertreten, wenn wir noch leis- tungsfähiger werden und die begonnene Kostenoptimierung vorantreiben. Für die FMH schliesslich steht das Jahr 2012 sicher für eine starke politische und mediale Präsenz. Sie wird als unausweichlicher Akteur im schweizerischen Gesundheitswesen wahrgenommen.

In dieser besinnlichen Zeit denkt man wieder an die Weihnachtswünsche, die Hoffnung auf eine friedliche Welt, auf Versöhnung und Harmonie. Man setzt sich neue Ziele, die es gemeinsam zu erreichen gilt. Diejenigen, die einen das letzte Jahr über geleitet haben, aber noch nicht erreicht sind, werden weiterverfolgt. Wie auch immer, das Generalsekreta-

\section{Für die FMH steht das Jahr 2012 für eine starke politische und mediale Präsenz. Sie wird als unausweichlicher Akteur im schweizerischen Gesund- heitswesen wahrgenommen.}

riat wird die grossen Herausforderungen, die sich im Gesundheitswesen heute und morgen stellen und im Bewusstsein der Veränderungen, die die Erfüllung der Erwartungen mit sich bringt, seinen Auftrag erfüllen. Wir setzen unsere interne Reform und unsere Professionalisierung so weit wie möglich fort. Wir stehen dem neuen Zentralvorstand weiterhin bestmöglich zur Seite, um ihn bei seinen Aufgaben zu unterstützen und zu entlasten. Als Kompetenzzentrum stellen wir uns weiter in den Dienst aller Mitglieder, der kantonalen Ärztegesellschaften und der Fachgesellschaften sowie der Partner der FMH. Weiter wird die Gelegenheit genutzt, die Bedürfnisse der Mitglieder zu prüfen, um ihre Erwartungen bestmöglich erfüllen und ihre Interessen optimal vertreten zu können. Bei all diesen Anstrengungen verlieren wir unser aller Interesse nicht aus den Augen: das Wohl der Patientinnen und Patienten.

So geht das Jahr 2012 mit einer kritischen und positiven Rückschau auf die vergangenen Monate und einem realistischen Ausblick auf das kommende Jahr zu Ende. In der Hoffnung, dass Sie diese Gedanken in Gelassenheit zum Jahresende begleiten, schliesse ich mich den Glückwünschen des Generalsekretariats an und wünsche Ihnen und Ihren Lieben schöne Festtage.

Anne-Geneviève Bütikofer, Generalsekretärin der FMH 